\title{
Modelling of advanced three-ion ICRF heating and fast ion generation scheme for tokamaks and stellarators.
}

\author{
J.M. Faustin ${ }^{1}$, J.P. Graves ${ }^{1}$, W.A. Cooper ${ }^{1}$, S. Lanthaler ${ }^{1}$, L. Villard ${ }^{1}$, D. Pfefferlé ${ }^{2}$, J. Geiger ${ }^{3}$, \\ Ye. O. Kazakov ${ }^{4}$ and D. Van Eester ${ }^{4}$ \\ ${ }^{1}$ Ecole Polytechnique Fédérale de Lausanne (EPFL), Swiss Plasma Center (SPC), CH-1015 \\ Lausanne, Switzerland \\ ${ }^{2}$ Princeton Plasma Physics Laboratory (PPPL), Princeton 08540 NJ, USA \\ ${ }^{3}$ Max-Planck Institut für Plasmaphysik, D-17491 Greifswald, Germany \\ ${ }^{4}$ Laboratory for Plasma Physics, LPP-ERM/KMS, EUROfusion Consortium member, TEC \\ Partner, Brussels Belgium
}

\begin{abstract}
Absorption of Ion-Cyclotron Range of Frequencies (ICRF) at the fundamental resonance is an efficient source of plasma heating and fast ion generation in tokamaks and stellarators. This heating method is planned to be exploited as a fast ion source in the Wendelstein 7-X stellarator. The work presented here assesses the possibility of using the newly developed three-ion species scheme [Y.O. Kazakov et al., Nucl. Fusion 55, 032001 (2015)] in tokamak and stellarator plasmas, which could offer the capability of generating more energetic ions than the traditional minority heating scheme with moderate input power. Using the SCENIC code, it is found that fast ions in the $\mathrm{MeV}$ range of energy can be produced in JET-like plasmas. The RF-induced particle pinch is seen to strongly impact the fast ion pressure profile in particular. Our results show that in typical high-density W7-X plasmas, the three-ion species scheme generates more energetic ions than the more traditional minority heating scheme, which makes three-ion scenario promising for fast-ion confinement studies in W7-X.
\end{abstract}

\section{Introduction}

Sustaining fusion reactions in a magnetic confinement device requires maintaining the plasma temperature to several $\mathrm{keV}$. In addition to the auxiliary plasma heating methods which are necessary to reach these temperatures, fusion-born $\alpha$ particles represent a substantial source of heating. The confinement of these particles for a duration comparable to their slowing down time is therefore of primary concern for the design of future fusion reactors. The quasi-isodynamic stellarator concept is seen as a suitable candidate for a future fusion reactor. One of the main objectives of Wendelstein 7$\mathrm{X}$ (W7-X), the recently started stellarator of this kind, is to demonstrate the good confinement energetic particles. An auxiliary source of fast ions with energies between 50 to $100 \mathrm{keV}$ is therefore required in $\mathrm{W} 7-\mathrm{X}$. Indeed, ions in that range of energy would mimic the behaviour of $\alpha$ particles in a reactor-size quasi-isodynamic stellarator, as discussed in Ref. [1].

Neutral Beam Injection (NBI) is one possible source of fast ions. A numerical assessment of the capacity of NBI to efficiently inject energetic ions in the plasma core of various $\mathrm{W} 7-\mathrm{X}$ equilibrium configurations was conducted in Ref. [1] and continued in Ref. [2]. This work led to the conclusions that the plasma density required for good central particle confinement prevents the beam to be deposited in the core plasma region. In addition, rapid losses of injected particles were observed in these simulations.

Ion-Cyclotron Range of Frequency (ICRF) waves is seen as an alternative because they have successfully been used for plasma heating and fast ion generation in tokamaks $[3,4]$ and stellarators $[5,6]$. The most used ICRF scenario in fusion devices is known as the minority heating $(\mathrm{MH})$ scheme [7]. Applied for example in typical Deuterium JET tokamak plasmas with moderately low Hydrogen concentration $(\sim 5 \%)$ and RF power $(\sim 3 \mathrm{MW})$, this scheme generates fast minority ions with energies above $100 \mathrm{keV}$. The ICRF system for W7-X is currently under development [8] and is envisaged as a possible substantial source of fast ion generation in W7-X with energies within the range of $50-100 \mathrm{keV}$. The possibility of using fundamental hydrogen minority heating in W7-X has been discussed in [9]. It was found that under relevant ICRF conditions (spatially lo- 
calised antenna and 1.5 MW of input power) significant loss of particles are observed and the fast ion tail, usually found in axisymmetric tokamak scenarios, is almost indistinguishable from the bulk plasma. Even though the particle losses were seen to significantly decrease in simulations by the inclusion of a radial electric field originating from the ambipolar diffusion of background electrons and ions, the formation of a fast ion tail remains hindered by the high plasma density.

An important aspect to take into account in the elaboration of efficient ICRF fast ion generation scenarios is the wave's electric field polarisation at the resonance position. In typical minority heating schemes, the wave polarisation is dictated by the plasma ion species and limits the amount of coupled power to the minority ions as discussed in Ref. [10]. In the latter work, the three-on species scheme was presented as a scenario that optimises the wave polarisation at the resonant species position and presents tremendous potential for fast ion generation with moderate input $\mathrm{RF}$ power. In the work presented here, we first report some modelling performed with the SCENIC package of the threeion species scheme applied to axisymmetric tokamak plasma. This scheme is then applied to a W7-X configuration in order to assess the possibility of using it for fast ion generation in W7-X.

This paper is organised as follows. The principle of the three-ion species scheme is briefly discussed in section (2). Numerical results obtained with the full wave code LEMan applied to a axisymmetric configuration are presented and display the key features of this ICRF scheme. In section (3), simulations obtained with the SCENIC package applied to an axisymmetric configuration are presented. The effect of varying the ICRF antenna phasing on the self-consistent ${ }^{3} \mathrm{He}$ ions distribution function is addressed. The possibility of generating fast ions with the three-ion species scheme applied to a high-mirror W7-X configuration is discussed in section (4). Conclusions are drawn in section (5).

\section{The three-ion species scheme}

In this section, we review the working principles of the three-ion species scheme. Propagation and absorption of fast magnetosonic waves depend on the number of ion species present in a plasma and their relative concentrations. Indeed, the latter dictate the behaviour of the plasma dielectric tensor and hence of the local fast wave dispersion relation. If one defines as $n_{\perp}$ and $n_{\|}$ the refractive indices in the perpendicular and parallel direction to the magnetic field lines, $\epsilon_{R}, \epsilon_{L}$ and $\epsilon_{S}$ the dielectric tensor elements as given by Stix in Ref. [7], the dispersion relation for the fast wave reads:

$$
n_{\perp}^{2}=\frac{\left(\epsilon_{R}-n_{\|}^{2}\right)\left(\epsilon_{L}-n_{\|}^{2}\right)}{\left(\epsilon_{S}-n_{\|}^{2}\right)} .
$$

In plasmas with two ion species with different chargeto-mass ratio the so-called mode conversion layer can be established. This is the spatial region bounded by the ion-ion hybrid resonance (defined by the relation $\left.\epsilon_{S}=n_{\|}^{2}\right)$ and the L-cutoff $\left(\epsilon_{L}=n_{\|}^{2}\right)$. The position of the mode conversion layer in a plasma is mainly dependent on the ion species concentration, and it has been traditionally used for localized electron heating with ICRF waves (see, e.g. Ref. [11]).

As explained in Ref. [10], the idea behind three-ion species heating scenario is to bring the mode conversion layer close to the cyclotron resonance of the third ion species. Since the left-hand electric field component $E^{+}$, which is responsible for the acceleration of thermal ions, is enhanced at the mode conversion layer, an efficient wave absorption by third ion species becomes possible at a very low minority concentration (\%olevel). The prerequisite for maximising the absorbed power on the third ion species is then to find the appropriate plasma mixture such that the L-cutoff is located close to the fundamental cyclotron resonance position of third ion species.

A typical JET-like plasma equilibrium is initially chosen in order to illustrate the fundamentals of the threeion species scheme. Wave propagation and absorption is computed with the LEMan code $[12,13]$, which implements a warm plasma dielectric tensor expanded to the $0^{\text {th }}$ order in Larmor radius. The LEMan code is therefore suited to model ICRF heating scenarios, for which fundamental cyclotron absorption is the dominant mechanism of wave damping. Even though LEMan is not able to model mode conversion phenomena and second harmonic absorption, the application of LEMan to model three-ion scenario is justified. Earlier fullwave modelling with TORIC [14], EVE [15] and TOMCAT [16] codes predicted that cyclotron absorption by ${ }^{3} \mathrm{He}$ ions is the dominant channel of RF power absorption and only a small fraction of power is absorbed by electrons, even though there are mode conversion layers present in the plasma. It is worth mentioning that the SCENIC package (which comprises the LEMan code, and used in the next sections) allows the inclusion of finite-orbit width effects when evaluating the distribution function of ICRF-heated ${ }^{3} \mathrm{He}$ ions. This is an important extension of the work presented here, in comparison with the earlier modeling of the three-ion species scenario.

The plasma modelled here consists mainly of a mixture of hydrogen and deuterium. The third species is 


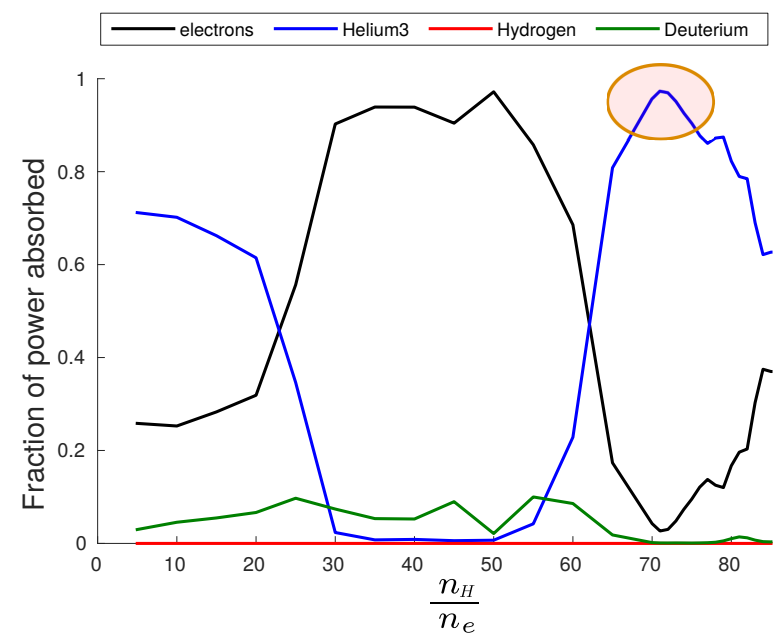

Figure 1 - Fraction of power deposited on each species for varying $\mathrm{H}$ concentration as calculated by the LEMan code $\mathrm{X}\left[{ }^{3} \mathrm{He}\right]=0.1 \%$. This code implements a warm plasma dielectric tensor expanded to the $0^{t h}$ order in Larmor radius.

${ }^{3} \mathrm{He}$ with a concentration as low as $0.1 \%$. The electron density and temperature profiles used result from analytic estimates and read: $n_{e}=\left[3.6 \times\left(1-\rho^{2}\right)+0.4\right] \times$ $10^{19} \mathrm{~m}^{-3}, T_{e}=\left[3 \times\left(1-\rho^{2}\right)^{3 / 2}+1\right] \mathrm{keV}$ where $\rho$ is the normalised minor radius. The magnetic axis is located at $R_{0}=2.96 \mathrm{~m}$ and the central magnetic field strength is $B_{0}=3.2 \mathrm{~T}$. We model the JET ICRF spectrum with a single toroidal wave mode number $\left(n_{\varphi}=27\right)$ which is propagating in the plasma at a frequency $f=32.5 \mathrm{MHz}$. The choice of frequency yields an on-axis ${ }^{3} \mathrm{He}$ fundamental resonance. Figure (1) shows the fraction of power absorbed by each species in the plasma when varying the $\mathrm{H}$ concentration for this test case. A peak of ${ }^{3} \mathrm{He}$ absorption clearly appears around $X[H]=\frac{n_{H}}{n_{e}} \simeq 70 \%$ which is in accordance with optimal mix calculations established with the TOMCAT code [16] as seen in Fig. 1(b) of Ref. [10].

The full-wave code LEMan is used to compute the dispersion relation given by eq. (1) which is showed in Fig. (2). The plasma mix in this case is $X[H]=$ $68 \%, X[D]=31.8 \%, X\left[{ }^{3} \mathrm{He}\right]=0.1 \%$. As seen in Figs. (2) the ${ }^{3}$ He fundamental cyclotron resonance position in the vicinity of the L-cutoff. Consequently and as seen in Fig. (3) the $\left|E^{+}\right|$electric field component (that rotates in the same direction of the ions) is the strongest very close the ${ }^{3} \mathrm{He}$ fundamental cyclotron resonance position. These features confirm that this configuration is favourable for the three-ion species scheme. Figure (1) also suggests that in the absence of other impurities, only electrons absorb a significant part of the RF

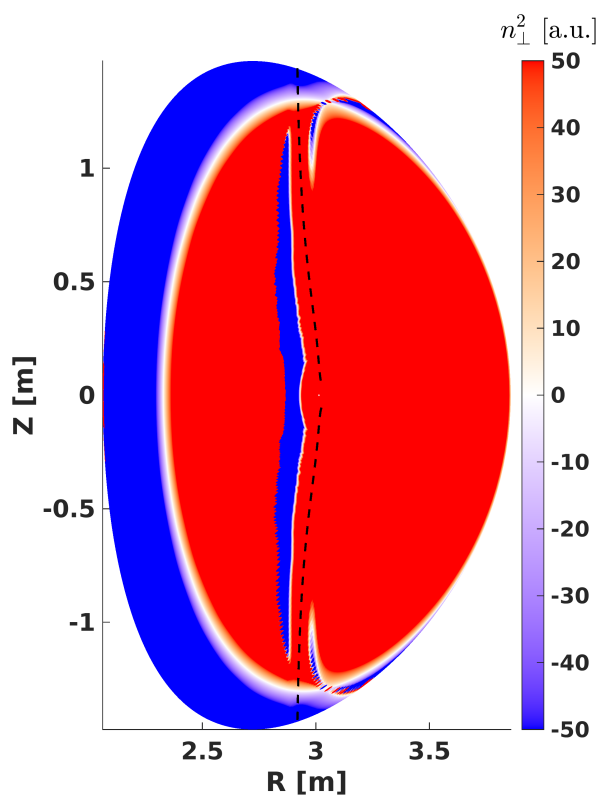

Figure 2 - Local dispersion relation given by eq. (1) $\left(n_{e 0}=4 \times 10^{19} \mathrm{~m}^{-3}, T_{0}=4 \mathrm{keV}\right), n_{\varphi}=27, \mathrm{X}[\mathrm{H}]=70 \%$ and $\mathrm{X}[3 \mathrm{He}]=0.1 \%)$. Evanescent and propagative regions respectively appear in blue and red. Cutoffs appear as white lines at the border of these regions. The black dashed lines show the fundamental ${ }^{3} \mathrm{He}$ resonant position.

power. As discussed in Ref. [10], an efficient absorption of $\mathrm{RF}$ power by ${ }^{3} \mathrm{He}$ ions was computed with the TORIC, EVE and TOMCAT codes. Therefore a scan in ${ }^{3} \mathrm{He}$ concentration is performed with the LEMan code and is shown in Fig. (4). It is important to mention that the LEMan code is missing the power absorption by electrons via Transit Time Magnetic Pumping [7]. Therefore it expected that the LEMan code underestimates the electron damping in scenarios where this branch is expected to dominate, typically for two-ion species scenarios with very low or high minority concentration. However, Fig. (4) is in good agreement with results from Ref. [10]: ${ }^{3} \mathrm{He}$ concentration for maximal power absorption is around $0.1 \%$.

\section{Two-dimensional modelling: JET-like axisymmetric plasma}

In this section, the SCENIC [17] package is applied to resolve fast ion distribution functions using the D$\left({ }^{3} \mathrm{He}\right)-\mathrm{H}$ ICRF scenario applied to a JET-like axisymmetric plasma. The VMEC/ANIMEC code [18] is used to construct an ideal MHD equilibrium based on typical JET tokamak plasma geometry and the density and temperature profiles mentioned in the previous section. 


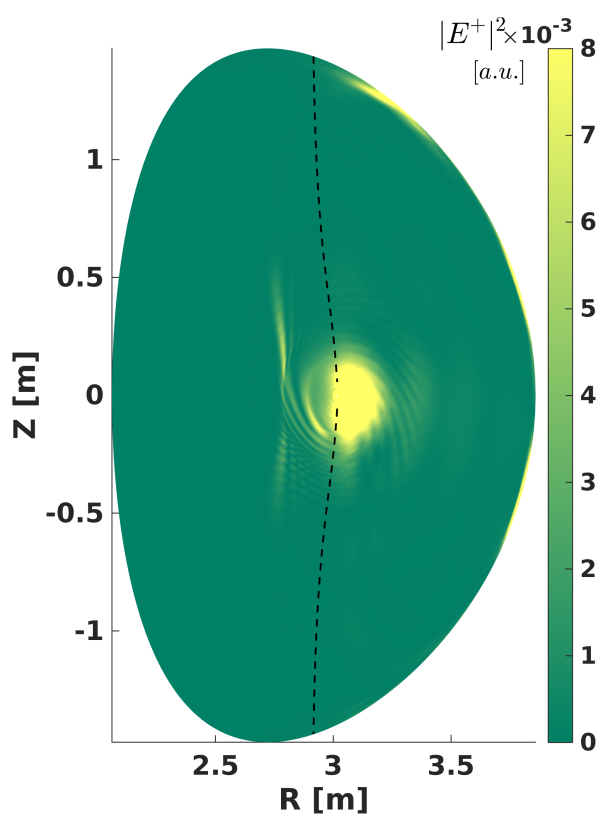

Figure 3 - Amplitude (in arbitrary units) of the electric field components rotating with the ions $\left(n_{e 0}=\right.$ $\left.4 \times 10^{19} \mathrm{~m}^{-3}, T_{0}=4 \mathrm{keV}\right), n_{\varphi}=27, \mathrm{X}[\mathrm{H}]=70 \%$ and $\mathrm{X}[3 \mathrm{He}]=0.1 \%)$. The black dashed lines show the fundamental ${ }^{3} \mathrm{He}$ resonant position.

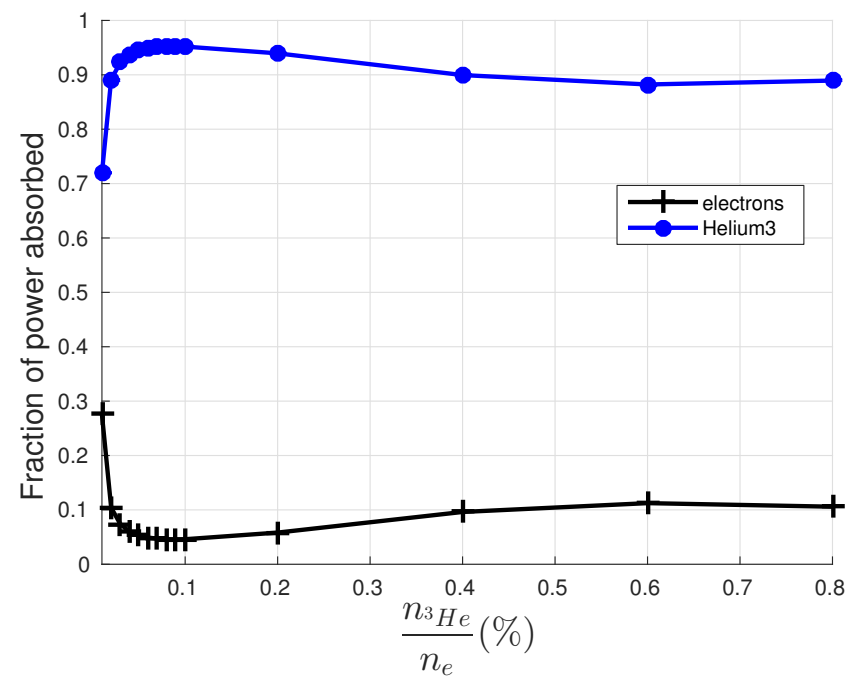

Figure 4 - Fraction of absorbed power on ${ }^{3} \mathrm{He}$ and electrons with varying ${ }^{3} \mathrm{He}$ concentration, $n_{e 0}=4 \times$ $\left.10^{19} \mathrm{~m}^{-3}, T_{0}=4 \mathrm{keV}\right), n_{\varphi}=27$ and $\mathrm{X}[\mathrm{H}]=70 \%$.

We will apply two different antenna phasings. The phasing has been shown to play an important role in particular on the resonant ion orbits due to the RF particle pinch. This effect has been discussed in [19,20], and experimental observations have been reported in [21,22]. The direction of the RF-pinch (inward or outward) can be controlled by the use of a toroidally asymmetric excitation spectrum. The effect of this phasing on the energy range reached by the resonant ions and the type of orbits are described next.

\subsection{Fast ion distribution function calcu- lation}

The interfacing of the codes composing the SCENIC package aims at solving iteratively the equilibrium state, the wave deposition, and the ${ }^{3} \mathrm{He}$ ion distribution function, until a self-consistent converged solution is obtained. The MHD equilibrium and the dielectric tensor calculations require some knowledge of the moments of the resonant ion distribution function. The heated ion distribution function usually develops a fast tail and therefore cannot be entirely modelled as a Maxwellian distribution. As described in more detail in Ref. [17], the resonant ion distribution function is split into thermal and fast components, which are respectively fitted onto a Maxwellian and a bi-Maxwellian model [23]. In the simulations presented here, the fast ion tail represents the most significant part of the marker distribution. The thermal part is mainly comprised of particles that were numerically re-injected in the plasma in a thermal state after having crossed the last closed flux surface. Considering the fraction and the energy range of the fast ions, it is assumed that only the fast component of the distribution significantly affects the plasma equilibrium and dielectric tensor. Therefore, only this component has been taken into account in the iteration scheme. Saturated solutions have been obtained for the antenna phasings most relevant for typical JET ICRF scenarios: dipole $\left(n_{\varphi}= \pm 27\right.$ ) and $+90^{\circ}$ (co-current travelling wave: $n_{\varphi}=-13$ ). Figure (5) shows that the mean energy per ${ }^{3} \mathrm{He}$ particle saturates with time for each phasing after a few iterations, indicating a converged self-consistent distribution function is obtained. The converged ${ }^{3} \mathrm{He}$ distribution functions for the two antenna phasings obtained with SCENIC for $3 \mathrm{MW}$ of ICRF power are shown in Fig. (6). It is seen that fast ions in the $\mathrm{MeV}$ range are generated in both cases. The distribution function of a typical minority heating (MH) scenario (deuterium rich plasma with 5\% hydrogen minority) with the same equilibrium profiles and input power conditions is shown for comparison. The significant potential of generating fast ions with moderate ICRF power clearly appears. Simulation results obtained with the AORSA and CQL3D codes [25] for comparable JET plasma parameters also indicate the generation of a large fraction of $\mathrm{MeV}$ ions with the threeion species scheme. 


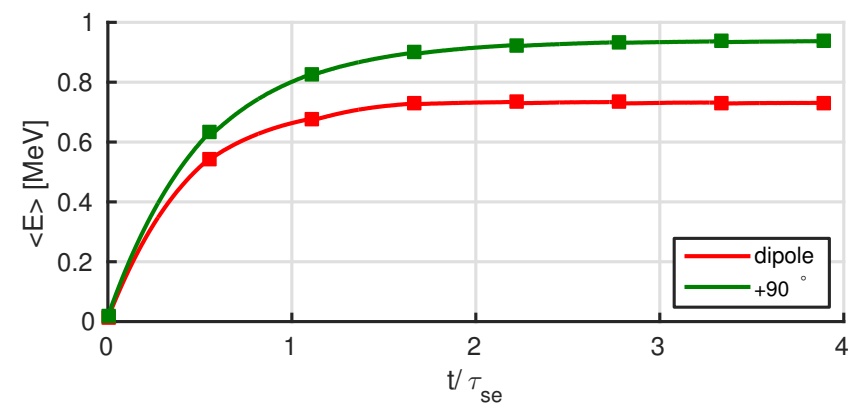

Figure 5- Mean energy per particle as a function of time normalised to the slowing down time on electrons [24] for ions at $1 \mathrm{MeV}: \tau_{s e}=0.18 \mathrm{~s}\left(P_{R F}=\right.$ $3 \mathrm{MW}, X[3 \mathrm{He}]=0.1 \%, n_{e 0}=4 \times 10^{19} \mathrm{~m}^{-3}, T_{0}=$ $4 \mathrm{keV}))$.

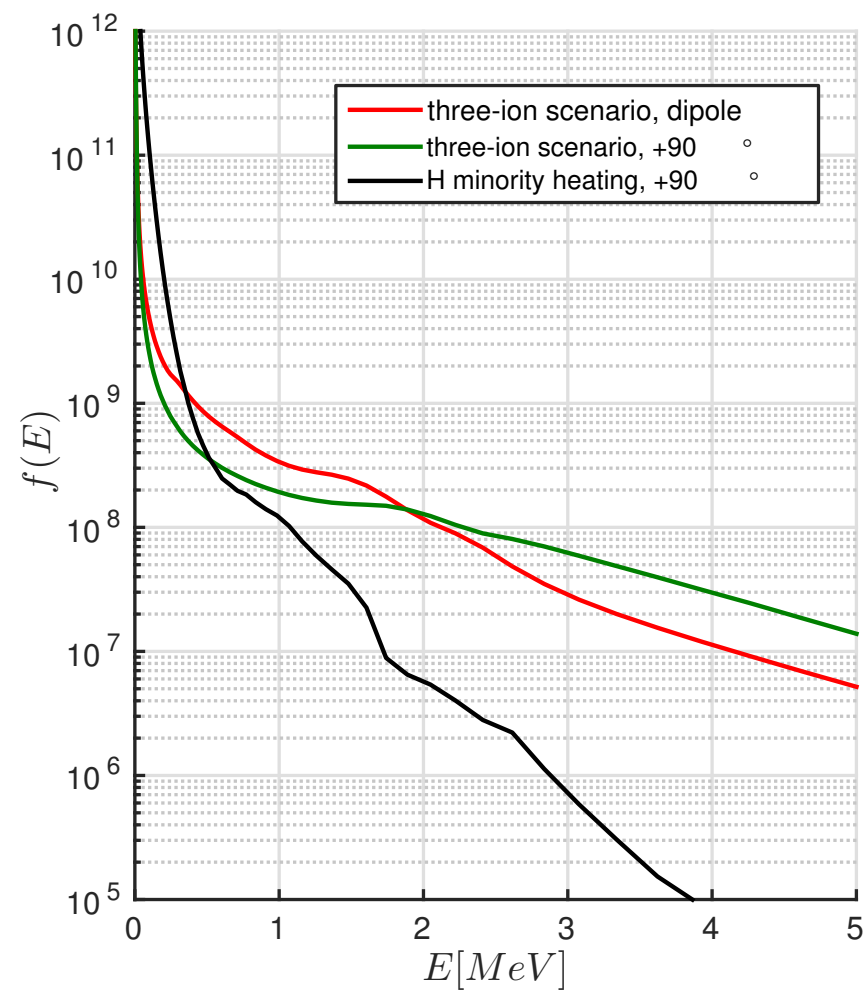

Figure 6 - Saturated energy distribution functions of ${ }^{3} \mathrm{He}$ ions for dipole and $+90^{\circ}$ phasings using three-ion species scheme $\left(X\left[{ }^{3} \mathrm{He}\right]=0.1 \%\right)$, and $+90^{\circ}$ phasing using a minority scheme $(X[H]=5 \%)$ for JET-like conditions $\left(P_{R F}=3 \mathrm{MW}\right)$.

\section{$3.2 \quad$ RF-pinch effect}

It is seen from Figs. (5) and (6) that using $+90^{\circ}$ phasing allows the generation of a larger fast ion tail than for dipole phasing. This can be explained mainly by the combination of two effects. Firstly, the $+90^{\circ}$ phased wave features a lower $\left|k_{\|}\right|$value (which can be approxi-

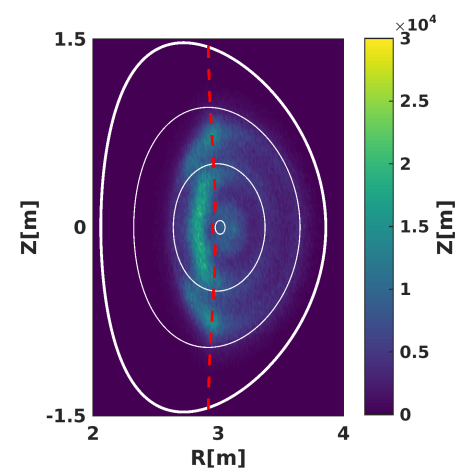

(a) Dipole

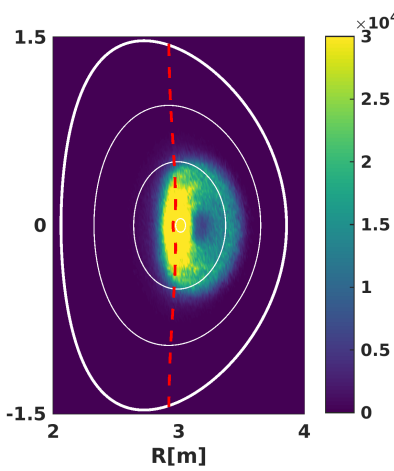

(b) $+90^{\circ}$
Figure 7 - Fast ion pressure profiles (Pa) for each investigated antenna phasing. The red dashed line represents the unshifted resonance position for ${ }^{3} \mathrm{He}$ ions.

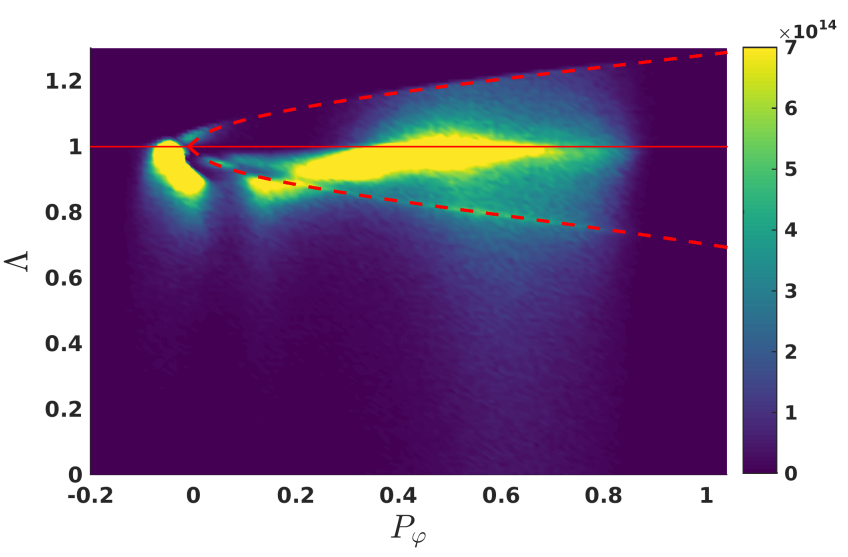

(a) Dipole

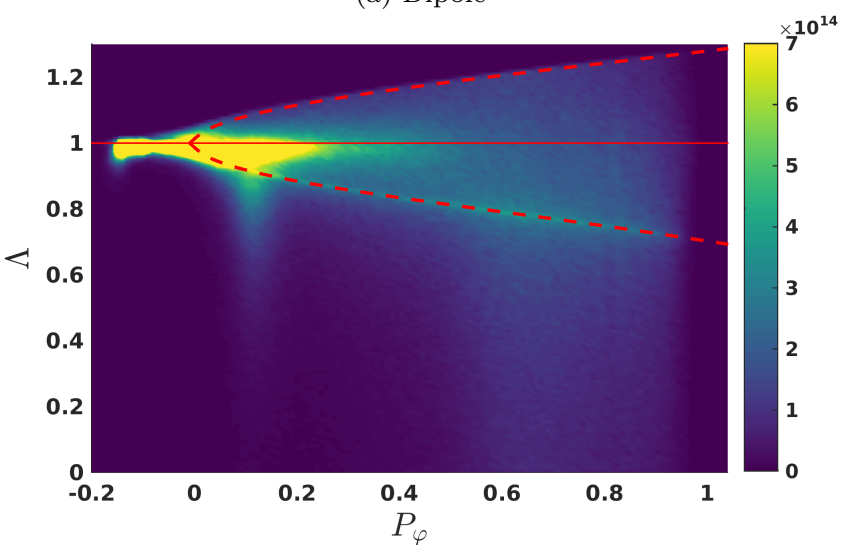

(b) $+90^{\circ}$

Figure 8 - Distribution (\#) of resonant ${ }^{3} \mathrm{He}$ particles in $\Lambda-P_{\varphi}$ plane. Note that $P_{\varphi}$ has been normalised for plotting convenience ( $q$ is the particles charge and $\psi_{0}$ is the poloidal flux on axis). The dashed and solid lines highlight respectively the trapped-passing boundary and the $\Lambda=1$ position. 
mated by $\left.\left|n_{\varphi}\right| / R\right)$ than the dipole phased wave. Therefore the absorption layer width is smaller in the $+90^{\circ}$ phasing case and consequently allows to achieve a higher RF power density than in the dipole case. Secondly, one has to consider the inward radial transport of resonant ions interacting with a co-current travelling ICRF wave. It was shown in Ref. [19] that RF wave-particle interaction not only result in kicks in the resonant particles' energy $\Delta E$ but also in the toroidal momentum $P_{\varphi}=-q \psi+m v_{\|} B_{\varphi} / B$, where $q, m$, and $v_{\|}$are respectively the particle's charge, mass and parallel velocity. The kick in the toroidal momentum reads:

$$
\Delta P_{\varphi}=\frac{n_{\varphi}}{\omega} \Delta E
$$

In eq. (2) $\omega$ is the RF wave angular frequency. In the VENUS-LEVIS code [26] which solves for the fast ion distribution function, the wave-particle interaction is resolved by Monte-Carlo operators that acts on the particles parallel and perpendicular velocities [17]. The kick in the parallel velocity reproduces the change in the toroidal momentum approximated by eq. (2). The magnetic moment of resonant particles is also affected by the interaction, such that on average resonant particles become trapped and their bounce tips align with the resonance layer. The change in the toroidal momentum subsequently results, on average, in a radial displacement of the trapped particles bounce tips along the resonance layer. It is seen from eq. (2), and the definition of $P_{\varphi}$, that co-current (resp. counter-counter) travelling waves induce an inward (outward) RF-pinch. The energy range reached by the fast ions (observable in Fig. (6)) suggests a strong RF-pinch effect. As seen in Fig. (7), $+90^{\circ}$ phasing produces strong on-axis peaking of the fast ion pressure, while dipole phasing spreads the fast ion pressure vertically along the resonance layer. In both cases, the pressure profile gives an insight into the classes of orbits that the resonant particles comprise. This can be addressed more precisely by collectively examining the resonant particles toroidal momentum $P_{\varphi}$ and the pitch angle variable $\Lambda=\frac{\mu}{E} B_{0}$ (here $\Lambda$ can be seen as a measure of a particles bounce tip position with respect to the on-axis magnetic field). In Refs. [27] and [28] these variables are used in order to draw a classification of guiding centre orbit types. Fig. (8) shows how the resonant particles are distributed in the $\Lambda-P_{\varphi}$ plane. The ICRF-induced particle trapping, and the resulting alignment of the particle bounce tips with the resonant layer causes the concentration of particles around the $\Lambda=1$ line. It is seen in Fig. (8b) that $+90^{\circ}$ phasing pushes the particles to the left-hand side of the $\Lambda-P_{\varphi}$ plane bringing the particles bounce tips closer to the magnetic axis. A significant fraction of the resonant particles are then able to escape the trapped region and enter the barely passing region of phase space. These highly energetic particles are consequently well confined in the plasma. The use of a $+90^{\circ}$ phased wave is as expected beneficial for the confinement of the RF accelerated fast ions. Moreover it is seen that for the dipole phasing simulation, more regions of the $\Lambda-P_{\varphi}$ diagram are populated. This can be explained by the fact that the wave-particle interaction with a dipole phased antenna is modelled by including two wave fields with a positive $\left(n_{\varphi}=27\right)$ and a negative mode number $\left(n_{\varphi}=-27\right)$ carrying the same fraction of ICRF power. This wave modelling technique, described in Ref. [15], is made possible by the weak coupling between the wave's toroidal mode numbers in axisymmetric configurations. The $n_{\varphi}=-27$ component acts in a similar way as the $+90^{\circ}$ phased wave, i.e. it induces an inward RF-pinch to particles, and thus is responsible for the population of barely passing particles seen in Fig. (8a). On the other hand, the $n_{\varphi}=27$ component induces an outward RF-pinch of particles which causes an increase in the trapped particle population. In addition, this pinch effect tends to push resonant particles towards the right-hand side of the $\Lambda-P_{\varphi}$ plane causing the generation of trapped particles with large orbit width. These orbits potentially cross the last closed surface resulting in a higher particle loss rate compared to the $+90^{\circ}$ case. This combined effect of RF-pinch and finite orbit width on the distribution function in phasespace, and in particular on the lost fraction, shows the importance of accounting for finite orbit width in simulations of the three-ion species scheme. Simulations with a $-90^{\circ}$ phased wave, corresponding to $n_{\varphi}=13$ were also conducted. However the significant particle loss rate produced by the outward RF pinch and the large orbit width brought difficulties in the convergence of the distribution function.

\subsection{Collisional power transfer}

The guiding centre orbit following code VENUS-LEVIS [26] includes the Coulomb interactions between the fast ions and the background electrons and ions. Figure (9) shows the energy dependency of the interaction time between ${ }^{3} \mathrm{He}$ ions and background electrons, hydrogen and deuterium ions. It was seen in the previous section that the energy of the simulated ${ }^{3} \mathrm{He}$ ions are mostly above $1 \mathrm{MeV}$. In that energy range, slowing down on electrons is the dominant Coulomb interaction. In comparison, the pitch angle scattering process on background species is around two orders of magnitude slower. This also suggests that the fast ion tail of the distribution functions is highly anisotropic. The Coulomb interaction between the ${ }^{3} \mathrm{He}$ markers and the background species is computed by VENUS-LEVIS and relies on the derivation of the associated Monte Carlo operator based on Ref. [29]. 


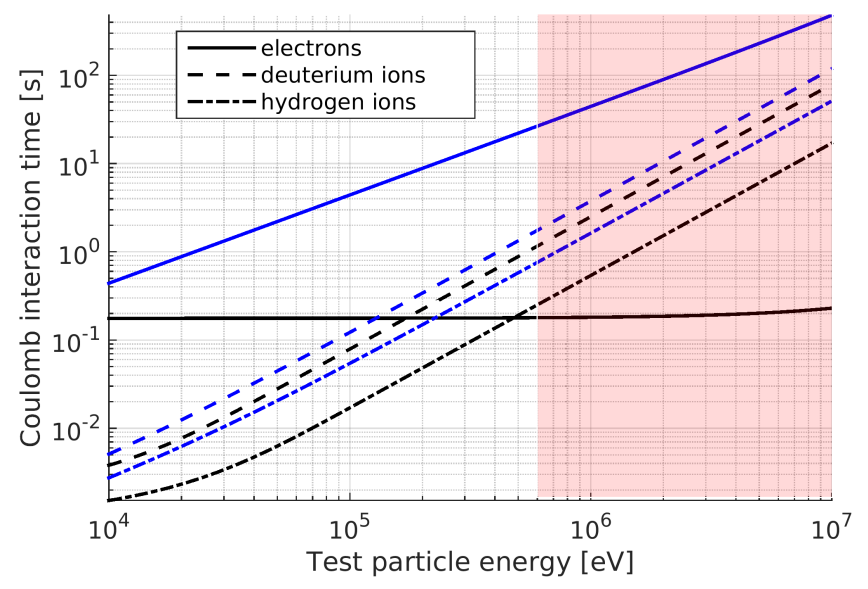

Figure 9 - Interaction time for slowing down (black lines) and pitch angle scattering (blue lines) of ${ }^{3} \mathrm{He}$ ions on background species [24]. The red area emphasises the relevant energy range for the simulations presented here.

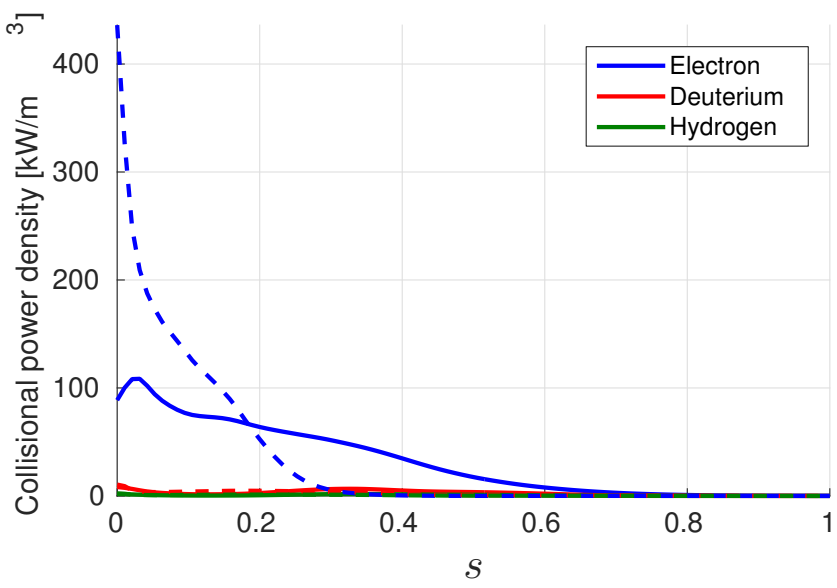

Figure 10 - Density of collisional power on each species. Dashed lines: $+90^{\circ}$ phasing; solid lines: dipole phasing.

The energy transfer corresponding to this interaction is evaluated assuming that the guiding centre ${ }^{3} \mathrm{He}$ particles collide with a static background species modelled as Maxwellian distribution functions. As expected, Figure (10) indicates that most of the collisional power resulting from slowing down interactions is transferred to background electrons. The collisional power deposition profile (Fig. (10)) and the ICRF power deposition profile (Fig. (11)) are noticeably affected by the RF-pinch effect on fast ions. It is seen that the ICRF power, and consequently the collisional power density transferred to electrons, are strongly peaked on axis for $+90^{\circ}$ phasing compared to the dipole case. Figure (10) already suggests that the three-ion heating scenario, especially if using $+90^{\circ}$ phasing, can be used in tokamak plasma

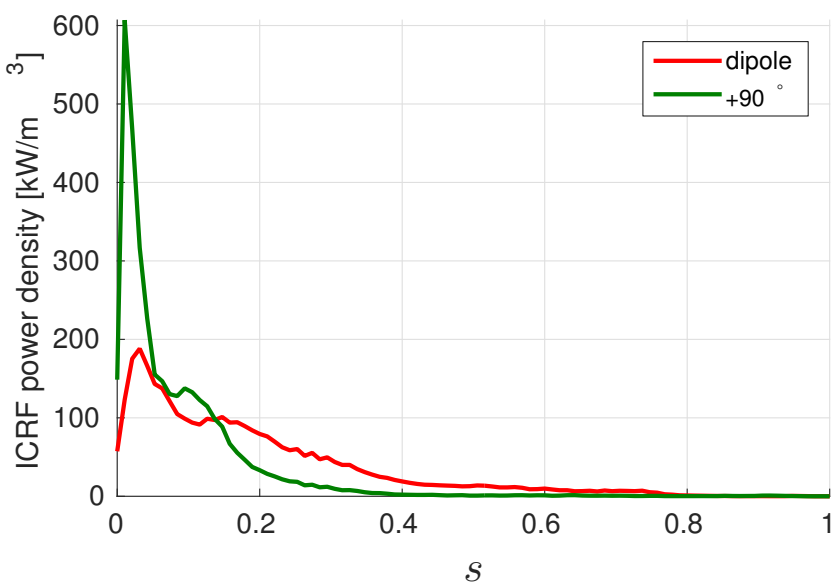

Figure 11 - Density of ICRF power on the ${ }^{3} \mathrm{He}$ ions for each investigated antenna phasing.

experiments to peak the central electron temperature. The fast ion simulations presented here could be coupled to transport calculations in order to estimate more quantitatively the impact of this scenario on the background thermal profiles. The peaking of the electron temperature profile can be beneficial for instance for enhancing the transport of impurities, such as Tungsten, out of the plasma core [30].

\section{Three-dimensional modelling: Wendelstein 7 -X plasma}

As previously mentioned, it was shown in Ref. [9] that minority heating scenarios may not be suitable for generating a large fast ion population mainly because of the high collisionality which prevents tail formation. In this section, the SCENIC code package is applied to a W7-X configuration in order to assess the possibility to use the three-ion species scheme as an alternative to minority heating. A high-mirror magnetic equilibrium is considered with the following density and temperature profiles expressed in terms of normalised toroidal flux $s: n_{e}=1.5 \times 10^{20}\left(0.9 \times\left(1-s^{10}\right)^{2}+0.1\right)\left[\mathrm{m}^{-3}\right]$ and $T_{e}=4 \times 10^{3}(1-s)[\mathrm{eV}]$. These profiles ensure that $\langle\beta\rangle \simeq 4 \%$ which is necessary to achieve a quasiomnigeneous equilibrium and consequently good fast particle confinement.

As suggested in Refs. [2,9], a radial electric field arising from an ion-root regime [31] is included in the following calculations in order to assist the confinement of the heated ions. A favourable plasma mix for the three-ion species scheme has been identified [10] as $X[H]=68 \%$, $X[D]=31.8 \%, X\left[{ }^{3} \mathrm{He}\right]=0.1 \%$. The corresponding dispersion relation is given in Fig. (12) for four different plasma cross-sections at toroidal angles $\varphi=0$, 

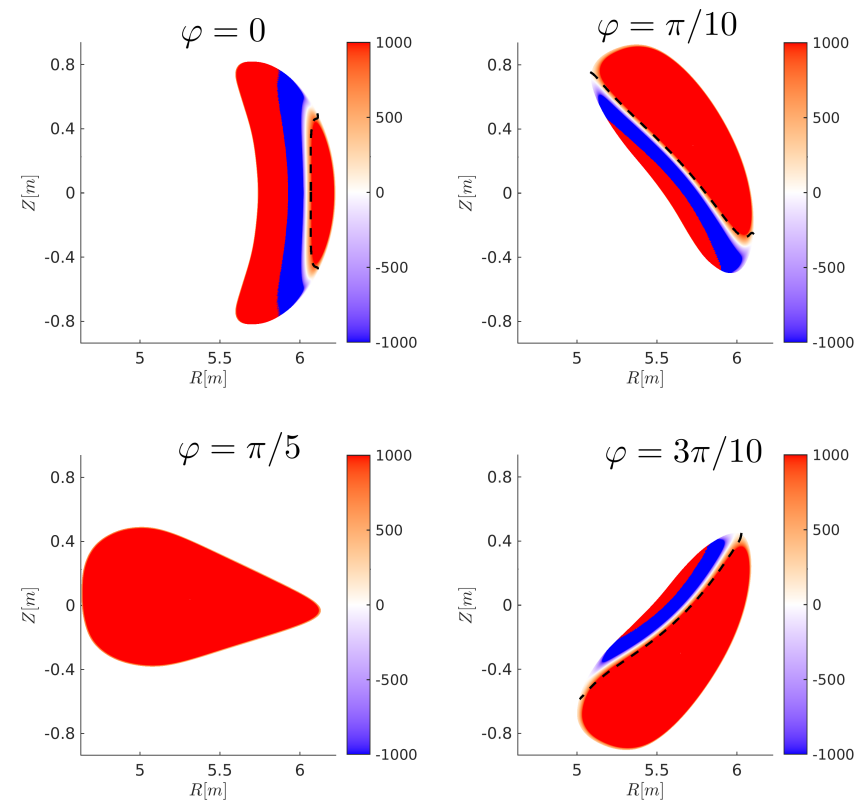

Figure 12 - Dispersion relation $\left(n_{\perp}^{2}\right)$ given by eq. (1) obtained with the three-ion species scheme applied to W7$\mathrm{X}$ with $\left.n_{e 0}=1.5 \times 10^{20} \mathrm{~m}^{-3}, T_{0}=4 \mathrm{keV}\right), \mathrm{X}\left[{ }^{3} \mathrm{He}\right]=0.1 \%$ and $\mathrm{X}[\mathrm{H}]=68 \%$. A localised antenna model is used to match the dimension of the actual system described in Ref. [8].

$\varphi=\pi / 10, \varphi=\pi / 5, \varphi=3 \pi / 10$. It seen that the Lcutoff is in the vicinity of the ${ }^{3} \mathrm{He}$ resonance position, as required by this scenario. This three-ion species scenario will be compared with a minority heating scheme that uses the same thermal profiles and MHD equilibrium. Previous [9] SCENIC calculations of minority heating in a D-rich W7-X plasma featured $\mathrm{H}$ minority with $X[H]=0.5 \%$. However, lowering $X[H]$ for increasing the efficiency of fast-ion generation is restricted by degeneracy between the resonance frequency for $\mathrm{H}$ minority and second harmonic $\mathrm{D}$ majority ions: $\omega_{c H}=2 \omega_{c D}$. In fact, in high-density plasmas of W7$\mathrm{X}$ most of the RF power is absorbed by $\mathrm{D}$ majority ions instead of $\mathrm{H}$ minority ions, reducing further the potential of this scenario for fast-ion studies. In contrast, using ${ }^{3} \mathrm{He}$ as a minority (as it is done in the following minority scheme) has an advantage of avoiding the degeneracy with the harmonic resonance for majority ions. Minority heating of ${ }^{3} \mathrm{He}$ in pure $\mathrm{H}$ or ${ }^{4} \mathrm{He}$ plasmas cannot be made effective at arbitrarily low ${ }^{3} \mathrm{He}$ concentrations. The efficiency of the singlepass plasma absorption strongly depends on the ${ }^{3} \mathrm{He}$ concentration. TOMCAT modelling suggests that the single-pass damping by ions is maximised at around $\mathrm{X}\left[{ }^{3} \mathrm{He}\right] \simeq 2 \%$ for $\mathrm{H}$ plasma and at around $\mathrm{X}\left[{ }^{3} \mathrm{He}\right] \simeq 4-5 \%$ in ${ }^{4} \mathrm{He}$ plasmas [32]. Further reduction of the ${ }^{3} \mathrm{He}$ con-

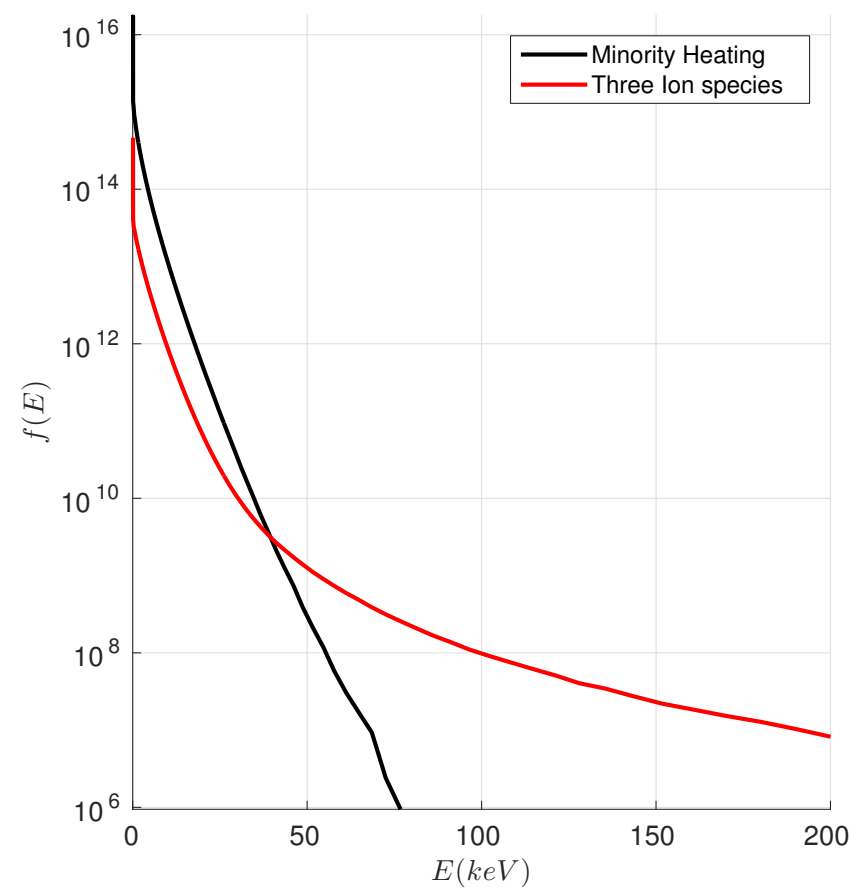

Figure 13 - Comparison of the ${ }^{3} \mathrm{He}$ ions distribution functions obtained with the minority heating $\left(X\left[{ }^{3} \mathrm{He}\right]=2 \%\right)$ and three-ion species schemes $\left(X\left[{ }^{3} \mathrm{He}\right]=0.1 \%\right)$ with $P_{R F}=1.5 \mathrm{MW}, n_{e 0}=1.5 \times$ $10^{20} \mathrm{~m}^{-3}, T_{0}=4 \mathrm{keV}$.

centration to the level of a few per mille is required to increase the efficiency of fast-ion generation in highdensity plasmas of W7-X.

Figure (13) compares the energy distribution functions obtained for both scenarios with $P_{R F}=1.5 \mathrm{MW}$. It is seen that no MeV-range ions appear in either of these scenarios, in contrast to tokamak calculations, because of the very high plasma density and limited amount of RF power available in W7-X. As a consequence, the contribution from the fast ion to the ideal MHD equilibrium and the wave propagation could not clearly be assessed and the usual iterative procedure of the SCENIC package could not be applied in the calculations presented in this section. It is nevertheless seen that the three-ion species scheme produces a fast ion tail which reaches energy range above $100 \mathrm{keV}$. In contrast, the minority heating distribution function is mostly thermal and the fast ion tail $(50-100 \mathrm{keV})$ is nearly non-existent. Precisely, the number of particles above $50 \mathrm{keV}$ obtained with the minority heating scenario $\left(X\left[{ }^{3} \mathrm{He}\right]=2 \%\right)$ reaches $3.36 \times 10^{14}$, whereas about 20 times more ions $\left(6.40 \times 10^{15}\right)$ are recorded in this range for the three-ion species scheme $\left(X\left[{ }^{3} \mathrm{He}\right]=0.1 \%\right)$. Particle orbits with energy below $50 \mathrm{keV}$ are largely affected by the $\boldsymbol{E} \times \boldsymbol{B}$ drift arising from the inclusion of 


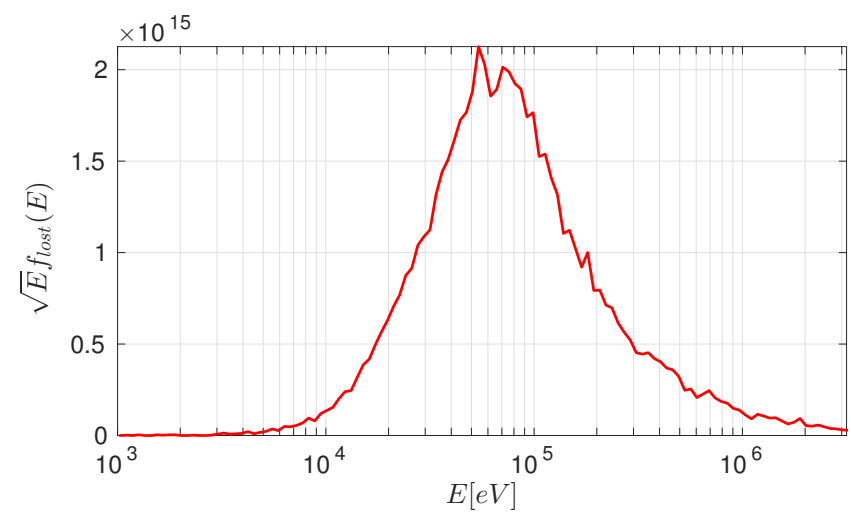

Figure 14 - Lost particle energy distribution function obtained with the three-ion species scheme.

the radial electric field. Therefore diffusion of thermal particles is the main loss channel in this case. Figure (14) shows the energy distribution of the recorded lost particles in the case where the three-ion species scheme is applied. It is seen that the energy range of lost particles is well above thermal energy. The effect of the radial electric field is therefore weaker on these energetic particles. The radial electric field is an important factor for the confinement of fast ions, generated with ICRF waves. If not optimized, this can result in losses of energetic ions as a result of their large orbit width. Furthermore, it seems that for the case of W7-X the typical thermal profiles, and the three-dimensional structure of the equilibrium significantly influence the tail formation process and consequently the heated ion distribution function in velocity space. More detailed analysis and conclusions on these aspects will be given in future work (e.g. studies on the optimal magnetic configuration for fast-ion generation).

\section{Summary and conclusions}

The work presented here shows modelling of the threeion species scheme that was first introduced in [10]. Two- and three-dimensional plasma equilibria were considered in order to asses the potential of the threeion species scheme in JET and W7-X plasmas. The SCENIC code package has been used and its suitability for the modelling of this scheme has been successfully demonstrated. It was shown that in a JET-like plasma, fast ions easily reach the $\mathrm{MeV}$ range of energy. As expected, varying the antenna phasing changes the direction of the RF-induced particle pinch. This effect is even more pronounced for the low ${ }^{3} \mathrm{He}$ concentration used in this scheme, implying large energy transfer during the resonant wave-particle interaction. It was seen that for co-current travelling ICRF waves, the inward pinch causes the orbits of resonant trapped ions to typically convert into passing orbits (which are better confined). In addition, the RF power density in the absorption layer is higher than for a dipole phased wave because the corresponding $k_{\|}$is smaller. Consequently, the establishment of the fast ion tail is assisted by the pinching of the fast ions towards the magnetic axis where the collisionality is lower and the wave absorption is higher. Estimation of the collisional power distribution indicates that co-travelling wave schemes may be used for the peaking of electron temperature profile (via localisation of the heated particles and Coulomb energy transfer) and may consequently help to increase the outward impurity transport. On the other hand, using a dipole phased wave applies in addition an outward pinch to resonant particles. Such a finite orbit width effect can cause the loss of resonant particles as they receive energy from the wave. The outward drift and the high loss rate reduce the mean energy of the resonant ion distribution and also causes the collisional power on the background species to be deposited in non-central plasma regions. Overall the fast ion distribution generated for both considered phasings show a more significant potential for fast ion generation than a typical minority heating scheme.

SCENIC simulations indicate that the three-ion species scheme in W7-X high density plasma would be more appropriate than a minority heating scheme for the generation of fast ions in this stellarator. However, the high density required to reach quasi-isodynamicity and therefore good fast particle confinement limits the range of energy reached by the resonant ions. It also appears that the tail formation process in $\mathrm{W} 7-\mathrm{X}$ is fundamentally affected by the three-dimensional nature of the equilibrium. The optimal magnetic configuration and favourable mechanisms for the formation of a significant fast ion tail in W7-X should be further investigated.

\section{Acknowledgements}

The authors would like to thank T. Johnson for very insightful discussions. This work has been carried out within the framework of the EUROfusion Consortium and has received funding from the European Union's Horizon 2020 research and innovation programme under grant agreement number 633053. The views and opinions expressed herein do not necessarily reflect those of the European Commission. The project was also supported in part by the Swiss National Science Foundation. The authors thank Dr. S. P. Hirshman for providing us with the VMEC code. Much of the numerical work was performed at the CSCS, Lugano, Switzerland, 
IFERC, Rokkasho, Japan and as well as the MarconiFusion cluster, hosted by CINECA/ENEA, Bologna, Italy. This work was supported by EPFL through the use of the facilities of its Scientific IT and Application Support Center.

\section{References}

[1] M. Drevlak, J. Geiger, P. Helander, and Y. Turkin. Fast particle confinement with optimized coil currents in the W7-X stellarator. Nuclear Fusion, 54(7):073002, 2014.

[2] J.M. Faustin, W.A. Cooper, J.P. Graves, D. Pfefferlé, and J. Geiger. Fast particle loss channels in Wendelstein 7-X. Nuclear Fusion, 56(9):092006, 2016 .

[3] D.F.H. Start, J. Jacquinot, V. Bergeaud, V.P. Bhatnagar, S.W. Conroy, G.A. Cottrell, S. Clement, G. Ericsson, L.-G. Eriksson, A. Fasoli, V. Fuchs, A. Gondhalekar, C. Gormezano, G. Gorini, G. Grosshoeg, K. Guenther, P.J. Harbour, R.F. Heeter, L.D. Horton, A.C. Howman, H.J. Jäckel, O.N. Jarvis, J. Källne, C.N. Lashmore Davies, K.D. Lawson, C.G. Lowry, M.J. Mantsinen, F.B. Marcus, R.D. Monk, E. Righi, F.G. Rimini, G.J. Sadler, G. Saibene, R. Sartori, B. Schunke, S.E. Sharapov, A.C.C. Sips, M.F. Stamp, M. Tardocchi, and P. van Belle. Bulk ion heating with ICRH in JET DT plasmas. Nuclear Fusion, 39(3):321, 1999.

[4] M. J. Mantsinen, L. G. Eriksson, M. GarcíaMuñoz, R. Bilato, Vl Bobkov, H. U. Fahrbach, J. M. Noterdaeme, and W. Schneider. Analysis of ICRF-accelerated ions in ASDEX upgrade. AIP Conf. Proc., 933:99-102, 2007.

[5] T. Mutoh, R. Kumazawa, T. Seki, T. Watari, K. Saito, Y. Torii, D. A. Hartmann, Y. Zhao, M. Sasao, M. Isobe, M. Osakabe, A. V. Krasilnikov, T. Ozaki, K. Narihara, Y. Nagayama, S. Inagaki, F. Shimpo, G. Nomura, M. Yokota, K. Akaishi, N. Ashikawa, P. de Vries, M. Emoto, H. Funaba, A. Fukuyama, M. Goto, K. Ida, H. Idei, K. Ikeda, N. Inoue, K. Itoh, O. Kaneko, K. Kawahata, S. Kado, A. Komori, T. Kobuchi, S. Kubo, S. Masuzaki, T. Morisaki, S. Morita, J. Miyazawa, S. Murakami, T. Minami, S. Muto, Y. Nakamura, H. Nakanishi, N. Noda, K. Nishimura, K. Ohkubo, N. Ohyabu, S. Ohdachi, Y. Oka, H. Okada, B. J. Peterson, A. Sagara, K. Sato, S. Sakakibara, R. Sakamoto, H. Sasao, M. Sato, T. Shimozuma, M. Shoji, S. Sudo, H. Suzuki, Y. Takeiri,
K. Tanaka, K. Toi, T. Tokuzawa, K. Tsumori, K. Y. Watanabe, T. Watanabe, H. Yamada, I. Yamada, S. Yamaguchi, K. Yamazaki, M. Yokoyama, Y. Yoshimura, Y. Hamada, O. Motojima, and M. Fujiwara. Ion heating and high-energy-particle production by ion-cyclotron heating in the large helical device. Phys. Rev. Lett., 85:4530-4533, Nov 2000 .

[6] S Murakami, a Fukuyama, T Akutsu, N Nakajima, V Chan, M Choi, S.C Chiu, L Lao, V Kasilov, T Mutoh, R Kumazawa, T Seki, K Saito, T Watari, $M$ Isobe, $T$ Saida, $M$ Osakabe, $M$ Sasao, and Lhd Experimental Group. A global simulation study of ICRF heating in the LHD. Nucl. Fusion, 46(7):S425-S432, July 2006.

[7] T H Stix. Fast-wave heating of a two-component plasma. Nuclear Fusion, 737, 1975.

[8] J. Ongena, A. Messiaen, D. Van Eester, B. Schweer, P. Dumortier, F. Durodie, Ye. O. Kazakov, F. Louche, M. Vervier, R. Koch, A. Krivska, A. Lyssoivan, M. Van Schoor, T. Wauters, V. Borsuk, O. Neubauer, O. Schmitz, G. Offermans, Y. Altenburg, C. Baylard, D. Birus, S. Bozhenkov, D. A. Hartmann, J. P. Kallmeyer, S. Renard, R. C. Wolf, and T. Fülöp. Study and design of the ion cyclotron resonance heating system for the stellarator Wendelstein 7-X. Phys. Plasmas, 21(6):061514, June 2014.

[9] J M Faustin, W A Cooper, J P Graves, D Pfefferlé, and J Geiger. ICRH induced particle losses in Wendelstein 7-X. Plasma Physics and Controlled Fusion, 58(7):074004, 2016.

[10] Ye.O. Kazakov, D. Van Eester, R. Dumont, and J. Ongena. On resonant ICRF absorption in threeion component plasmas: a new promising tool for fast ion generation. Nucl. Fusion, 55:032001, 2015.

[11] M.J. Mantsinen, M.-L. Mayoral, D. Van Eester, B. Alper, R. Barnsley, P. Beaumont, J. Bucalossi, I. Coffey, S. Conroy, M. de Baar, P. de Vries, K. Erents, A. Figueiredo, A. Gondhalekar, C. Gowers, T. Hellsten, E. Joffrin, V. Kiptily, P.U. Lamalle, K. Lawson, A. Lyssoivan, J. Mailloux, P. Mantica, F. Meo, F. Milani, I. Monakhov, A. Murari, F. Nguyen, J.-M. Noterdaeme, J. Ongena, Yu. Petrov, E. Rachlew, V. Riccardo, E. Righi, F. Rimini, M. Stamp, A.A. Tuccillo, K.-D. Zastrow, M. Zerbini, and JET EFDA contributors. Localized bulk electron heating with ICRF mode conversion in the JET tokamak. Nuclear Fusion, 44(1):33, 2004. 
[12] P. Popovich, W. A. Cooper, and L. Villard. A fullwave solver of the Maxwell's equations in 3D cold plasmas. Comput. Phys. Commun., 175(4):250 263, August 2006.

[13] N. Mellet, W. A. Cooper, P. Popovich, L. Villard, and S. Brunner. Convolution and iterative methods applied to low-frequency waves in 3D warm configurations. Comput. Phys. Commun., 182(3):570 589, March 2011.

[14] M Brambilla. Numerical simulation of ion cyclotron waves in tokamak plasmas. Plasma Physics and Controlled Fusion, 41(1):1, 1999.

[15] R.J. Dumont and D. Zarzoso. Heating and current drive by ion cyclotron waves in the activated phase of iter. Nuclear Fusion, 53(1):013002, 2013.

[16] D Van Eester and R Koch. A variational principle for studying fast-wave mode conversion. Plasma Physics and Controlled Fusion, 40(11):1949, 1998.

[17] M. Jucker, J. P. Graves, W. A. Cooper, N. Mellet, T. Johnson, and S. Brunner. Integrated modeling for ion cyclotron resonant heating in toroidal systems. Comput. Phys. Commun., 182(4):912-925, April 2011.

[18] W. A. Cooper, S. P. Hirshman, P. Merkel, J. P. Graves, J. Kisslinger, H. F G Wobig, Y. Narushima, S. Okamura, and K. Y. Watanabe. Three-dimensional anisotropic pressure free boundary equilibria. Comput. Phys. Commun., 180(9):1524-1533, September 2009.

[19] Liu Chen, J. Vaclavik, and G.W. Hammett. Ion radial transport induced by icrf waves in tokamaks. Nuclear Fusion, 28(3):389, 1988.

[20] T. Johnson, T. Hellsten, and L. G. Eriksson. Analysis of a quasilinear model for ion-cyclotron interactions in tokamaks. AIP Conf. Proc., 787(7):54-57, July 2005.

[21] L.-G. Eriksson, M. Mantsinen, D. Borba, a. Fasoli, R. Heeter, S. Sharapov, D. Start, J. Carlsson, a. Gondhalekar, T. Hellsten, and a. Korotkov. Evidence for a Wave-Induced Particle Pinch in the Presence of Toroidally Asymmetric ICRF Waves. Phys. Rev. Lett., 81(6):1231-1234, 1998.

[22] M. J. Mantsinen, L. C. Ingesson, T. Johnson, V. G. Kiptily, M.-L. Mayoral, S. E. Sharapov, B. Alper, L. Bertalot, S. Conroy, L.-G. Eriksson, T. Hellsten, J.-M. Noterdaeme, S. Popovichev, E. Righi, and A. A. Tuccillo. Controlling the Profile of IonCyclotron-Resonant Ions in JET with the WaveInduced Pinch Effect. Phys. Rev. Lett., 89:115004, Aug 2002.

[23] W.A Cooper, J.P Graves, S.P Hirshman, T Yamaguchi, Y Narushima, S Okamura, S Sakakibara, C Suzuki, K.Y Watanabe, H Yamada, and K Yamazaki. Anisotropic pressure bi-Maxwellian distribution function model for three-dimensional equilibria. Nucl. Fusion, 46(7):683-698, July 2006.

[24] J. D. Huba. NRL Plasma Formulary. Naval Research Laboratory, Washington, D.C., 2006.

[25] Ye. O. Kazakov et al. Plasma heating and generation of energetic ions with novel three-ion ICRF scenarios on Alcator C-Mod and JET tokamak facilities. $58^{\text {th }}$ APS-DPP Meeting (San Jose, CA), invited talk, NI3.00005, 2016.

[26] D. Pfefferlé, W.A. Cooper, J.P. Graves, and C. Misev. VENUS-LEVIS and its spline-Fourier interpolation of 3D toroidal magnetic field representation for guiding-centre and full-orbit simulations of charged energetic particles . Computer Physics Communications, 185(12):3127 - 3140, 2014.

[27] C. T. Hsu and D. J. Sigmar. Alpha-particle losses from toroidicity-induced Alfvén eigenmodes. Part I: Phase-space topology of energetic particle orbits in tokamak plasma. Physics of Fluids B, 4(6):14921505, 1992.

[28] L-G Eriksson and F Porcelli. Dynamics of energetic ion orbits in magnetically confined plasmas. Plasma Phys. Control. Fusion, 43(4):R145-R182, 2001.

[29] A.H. Boozer and G. Kuo-Petravic. Monte Carlo evaluation of transport coefficients. Phys. Fluids, $24(5): 851,1980$.

[30] E. Lerche, M. Goniche, P. Jacquet, D. Van Eester, V. Bobkov, L. Colas, C. Giroud, I. Monakhov, F.J. Casson, F. Rimini, C. Angioni, M. Baruzzo, T. Blackman, S. Brezinsek, M. Brix, A. Czarnecka, K. Crombé, C. Challis, R. Dumont, J. Eriksson, N. Fedorczak, M. Graham, J.P. Graves, G. Gorini, J. Hobirk, E. Joffrin, T. Johnson, Y. Kazakov, V. Kiptily, A. Krivska, M. Lennholm, P. Lomas, C. Maggi, P. Mantica, G. Mathews, M.-L. Mayoral, L. Meneses, J. Mlynar, P. Monier-Garbet, M.F. Nave, C. Noble, M. Nocente, I. Nunes, J. Ongena, G. Petravich, V. Petrzilka, T. Pütterich, M. Reich, M. Santala, E.R. Solano, A. Shaw, G. Sips, M. Stamp, M. Tardocchi, M. Tsalas, M. Valisa, 
and JET Contributors. Optimization of ICRH for core impurity control in JET-ILW. Nuclear Fusion, 56(3):036022, 2016.

[31] Y. Turkin, C. D. Beidler, H. Maaßberg, S. Murakami, V. Tribaldos, and A. Wakasa. Neoclassical transport simulations for stellarators. Physics of Plasmas, 18(2), 2011.

[32] Ye. O. Kazakov, D. Van Eester, J. Ongena, and T. Fülöp. Modelling of the ion cyclotron resonance heating scenarios for W7-X stellarator. AIP Conference Proceedings, 1580(1):342-345, 2014. 